Caligrama, Belo Horizonte, v. 23, n. 3, p. 99-121, 2018

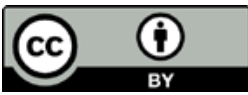

\title{
Être ou ne pas être post(-)coloniale : le cas de la littérature des intrangers
}

\author{
To Be or Not to Be Post(-)colonial: \\ The Case of the Literature of the Intrangers
}

\author{
Ioana Marcu \\ West University of Timisoara, Timișoara / Romênia \\ ioana.marcu@e-uvt.ro
}

Résumé :Dans l'espace francophone, parler de « littératures postcoloniales » représente un véritable défi. D'une part, il s'agit d'un courant littéraire envisagé avec méfiance, voire même réticence et hostilité, malgré les écrivains et les philosophes français et francophones cités comme précurseurs. De l'autre part, les caractéristiques éclectiques du corpus postcolonial donnent lieu à un rassemblement sous un même label d'œuvres littéraires protéiformes (aussi bien sur le plan de l'espace et de l'époque d'émergence que sur le plan des problématiques traitées et de la langue d'écriture); parallèlement, elles entraînent une mise à l'écart d'un ensemble littéraire hybride (à cause de l'histoire personnelle des écrivains et de leur langue d'expression). Dans notre contribution, nous nous proposons de voir si la littérature des intrangers, produite par les descendants d'immigrés maghrébins ou de harkis, présente vraiment les caractéristiques du corpus post(-)colonial. Nous fonderons notre analyse sur deux romans de l'extrême contemporain appartenant à deux écrivaines ayant connu un destin complètement différent : Un homme, ça ne pleure pas (2014) de Faïza Guène, chouchou des médias, cadette d'une famille originaire d'Algérie, née à Bobigny et ayant grandi dans la cité des Courtillières à Pantin, et Mohand le harki (2003) de Hadjila Kemoum, elle-même fille de harki.

Mots-clés : post(-)colonial, intrangers, hybride, harkis, immigration.

Abstract : In the French-speaking space, talking about "postcoloniales literatures" represents a real challenge. It is about a literary movement envisaged suspiciously, even reluctant and hostile, despite the French and Francophone writers and philosophers cited 
as being the precursors. On the other hand, the eclectic peculiarities of the postcolonial corpus give rise, at the same time, to a gathering under the same label of protean literary works, from the point of view of the space and of the period of emergence as well as the point of view of the problems treated and of the language of writing. Meanwhile, it also considers the hybrid corpus because of the personal history of the writers and of their language of expression. In our contribution, we investigate whether the literature of intrangers, produced by the descendants of Maghrebi immigrants or harkis, really presents the characteristics of the post(-)colonial corpus. We base our analysis on two novels of two contemporary female writers who knew a completely different destiny: Un homme, ça ne pleure pas (2014), of Faïza Guène, younger child of an Algerian family, born in Bobigny and raised in Courtillières, and Mohand le harki (2003), by Hadjila Kemoum, herself girl of harki.

Keywords : post (-) colonial, intrangers, hybrid, harkis, immigration.

La France et le monde francophone auraient raté le rendez-vous théorique important de la fin de siècle ? Est-ce parce que le mot postcolonial traîne encore du "colonial" ne serait-ce que phonétiquement, et que ses charges sémantiques sont encore lourdes ? Est-ce à cause de la guerre des mémoires qui concerne la gestion d'un passé commun ? Est-ce parce l'humanisme, ce "tout-homme", longtemps confisqué au seul profit du monde colonial blanc, s'est voulu seul modèle et seule perspective, excluant tout le divers du monde?

Zineb Ali-Benali et Françoise Simasotchi-Bronès

Quarante ans après la naissance du postcolonialisme aux ÉtatsUnis et une quinzaine d'années après l'introduction (particulièrement) timide des postcolonial studies en France, il est toujours exceptionnel (voire peut-être même osé) d'approfondir le corpus littéraire produit en langue française par des écrivains issus de la périphérie à partir d'une approche singulière, caractérisée "par sa pluridisciplinarité", "interrogeant l'histoire coloniale et ses traces jusque dans le monde contemporain" (MOURA, 2006). Le fait est d'autant plus problématique lorsque l'on s'intéresse à la littérature des intrangers, née de la volonté d'affirmation des individus dont on n'avait pas prévu l'irruption dans la société française - les descendants de ceux qui ont quitté le Maghreb (notamment l'Algérie) pour des raisons économiques (les immigrés) 
ou pour fuir les massacres (les harkis) dans le but de venir s'installer en France. Ces intrangers ont donc décidé un jour que le temps est venu pour sortir du silence auquel on avait condamné leurs parents, les souffre-douleur de la colonisation et du passage vers les indépendances, ceux qui ont choisi de se mettre à l'abri dans l'ancienne métropole colonisatrice afin d'avoir le droit à une vie meilleure. Les beurs et les enfants des harkis ont pris donc la parole pour faire connaître aux autres (notamment au public français) ce que signifie de vivre en marge de la société et à la périphérie l'espace (dans des banlieues, des bidonvilles, des camps), dans la pauvreté et la précarité, d'être sans cesse victime du racisme malgré sa naissance sur le territoire français. Par le biais de leurs productions littéraires, ils ont voulu également exhiber des bribes du passé douloureux de leurs géniteurs dans un pays mutilé par la colonisation, un ancien temps fait de souffrance, de désespoir, de rage, de souvenirs ensanglantés, un passé étouffé longtemps à l'intérieur des familles et, ce qui est plus bouleversant, au sein de la société.

Dans ce qui suit, nous nous proposons de voir, tout en nous appuyant sur les particularités des littératures postcoloniales, si le corpus littéraire appartenant aux écrivains nés en France à la suite du déplacement de leurs parents d'origine maghrébine ${ }^{1}$ est en état de porter le label postcolonial applicable le plus souvent à des écrits témoignant soit d' "une attitude de résistance contre la domination coloniale" (HARGREAVES, 2007, p. 25) et de contestation, soit d'une "[déconstruction] des codes coloniaux" (MOURA, 2000) ou d'une volonté de "dépister les traces [de l'histoire coloniale] jusqu'à notre époque" (MOURA, 1999, p. 173). Nous fondons notre étude sur deux romans s'attachant à des questions distinctes (le processus de formation d'un jeune issu d'une famille d'immigrés ; les conséquences dévastatrices de la guerre sur l'individu et sur ses proches), appartenant à deux écrivaines ayant elles-mêmes un parcours tout à fait singulier.

\footnotetext{
${ }^{1}$ Étant donné la nature différente du déplacement - économique ou sociale pour les immigrés et catastrophique pour les harkis -, il nous semble pertinent de ne pas parler d'une "littérature issue de l'immigration" mais plutôt d'une "littérature issue de la mobilité" maghrébine.
} 
Faïza Guène est sans doute le nom le plus médiatisé et le plus connu de la dernière vague de la littérature issue de l'immigration maghrébine. ${ }^{2}$ On la désigne "Sagan des cités" (FOHR, 2004), "Candide en banlieue" (ALILAT, 2004), "la sale môme qui écrit des best-sellers" (SUBTIL, 2006), "un ovni dans le monde des lettres" (GUENE, 2015), etc. Née dans la banlieue Nord de Paris, dans une famille d'origine algérienne, elle reste fidèle à ces "toxi-cités" (BELETRECHE, 2013) où elle continue à vivre. Découverte par son professeur de français, Boris Séguin, Guène publie son premier roman Kiffe kiffe demain chez Hachette Littératures en 2004. Un homme, ça ne pleure pas, auquel nous nous intéressons dans cette contribution, parait en 2014, chez Fayard. Le roman retrace quelques mois de la vie de Mourad, le seul fils de la famille Chennoun, qui vient de décrocher un poste de professeur de lettres en région parisienne. Tout en racontant son propre parcours, Mourad dessine les trajets contradictoires des autres membres de sa famille : d'un côté il y a Dounia, la fille aînée, en rupture avec la famille et avec tout ce qu'elle signifie (histoire personnelle, traditions, honneur, etc.) ; de l'autre côté il y a Djamilla, la mère, celle qui veille opiniâtrement au respect de l'héritage ancestral, et Mina, la cadette de la famille, qui prend admirablement la relève de sa mère ; il y a également le père, le personnage silencieux, effacé, qui n'arrive pas à jouir de son rôle de chef de famille, mais qui réussit finalement, à sa mort, à réunir les membres égarés du clan.

Quant à Hadjila Kemoum, l'auteure du deuxième roman de notre corpus, elle connaît un parcours beaucoup moins visible. Elle est née également dans une famille d'origine algérienne à la différence que son père a été harki. ${ }^{3}$ Son roman ("historique") Mohand le harki retrace l'histoire poignante d'une famille dont toute la vie a été ravagée par les accords d'Évian, le départ de l'armée française et l'ascension du FLN : les parents de Mohand sont torturés et tués par des fellagha; il s'engage alors dans l'armée à côté de la France ; lorsque l'indépendance du pays est déclarée, il doit fuir l'Algérie et réussit à s'embarquer pour la France avec sa famille grâce à des militaires français qui ont désobéi aux ordres reçus de leurs supérieurs. Mohand ne parle pas à ses enfants de manière

\footnotetext{
${ }^{2}$ Voir MARCU, I. La problématique de l'"entre(-)deux" dans la littérature des "intranger.e.s".

${ }^{3}$ Elle est à présent chef de projet dans une entreprise et présidente d'une association.

${ }^{4}$ Cette indication apparaît sur la page LinkedIn de l'auteure.
} 
directe de tout ce qu'il a vécu, de tous les massacres qu'il a vus; il ne veut pas les accabler et leur transmettre son propre déchirement. Il n'y a que quelques photos qui parlent à ses enfants de lui. Ce n'est qu'au moment où il commet l'irréparable et meurt que ses enfants comprendront le sacrifice de leur père. Ce "silence sur la guerre [...], [cette] volonté de ne pas lester les enfants d'un passé trop lourd et [...] [ce] désir de les projeter dans l'avenir" (KRAENKER, 2009, p. 223) détruit finalement une famille déjà anéantie par une conjoncture historique.

Après avoir dans un premier temps apporté brièvement quelques précisions sur les études postcoloniales et leur accueil en France, nous insisterons sur les indices extra et intratextuels qui nous permettent d'envisager les deux romans, et par extension, le corpus issu de la mobilité nord-africaine, tantôt comme "postcoloniaux" tantôt comme incompatibles avec cette épithète.

\section{Les études postcoloniales en tant que "chantier" (BARDOLPH, 2001, p. 59)}

La "bible" (DIOUF, 2006, p. 25) : Orientalism d'Edward Saïd publié en 1978. Un deuxième ouvrage de chevet : The Empire Writes Back. Theory and Practice in Post-Colonial Literature publié par Bill Ashcroft, Helen Tiffin et Gareth Griffiths en 1989..$^{5}$ La "holy trinity" (YOUNG, 1995, p. 153) ou le "noyau dur des études postcoloniales contemporaines" (DELLA FAILLE, 2012, p. 16) : Edward Saïd, Gayatri Chakrabarty Spivak et Homi K. Bhabha. Voilà les données constitutives des études postcoloniales.

Mais l'histoire de ces théories est beaucoup plus complexe que cela. Elles sont nées dans les années 1960-70 lorsque des intellectuels originaires des anciennes colonies anglaises (notamment de l'Inde), après être arrivés dans des universités anglophones (dans des départements de langues et de littératures), commencent à s'intéresser aux subalternes et à proposer une "relecture de l'histoire du passé colonial, [...] une critique

\footnotetext{
${ }^{5}$ Pour Alec G. Hargreaves, c'est à partir de la publication de cet ouvrage que les “" postcolonial studies' prennent leur essor institutionnel pour devenir un point nodal des recherches en sciences humaines au cours des années 1990" (HARGREAVES, 2007, p. 25). Et à l'auteur de continuer: "La parution de The Empire Writes Back en 1989 coïncide avec l'effondrement du rideau de fer et la fin de la Guerre froide, entraînant de profonds changements dans les paradigmes dominants dans les sciences humaines et sociales" (HARGREAVES, 2007, p. 25).
} 
du néocolonialisme, $[\ldots]$ une réévaluation de ses conséquences actuelles et aussi $[\ldots]$ une remise en cause de toute l'anthropologie occidentale, décrite comme coloniale" (CONTE, 2010, p. 73). Progressivement, les postcolonial studies s'ouvrent vers "les diasporas, les migrants, les réfugiés", proclament "la pluralisation culturelle des sociétés du monde dans sa globalité, soit au Sud comme au Nord" et problématisent les"identités culturelles dans le monde,lesquelles ne sont considérées ni fixes, ni pures, ni nettement circonscrites par les contours des Étatsnations" (BENESSAIEH, 2010). Pour Jean-Marc Moura, l'importance de l'approche postcoloniale dans les études littéraires réside dans leur effort de "rendre justice aux conditions de production et aux contextes socio-culturels dans lesquels s'ancrent ces littératures [issues des anciens pays colonisés]" et dans leur volonté de ne pas être envisagées comme "de simples extensions de la littérature européenne qui n'auraient pas à être situées pour être comprises” (MOURA, 2006).

Les théories postcoloniales s'inspirent notamment des travaux des écrivains et philosophes francophones. Léopold Sédar Senghor, Frantz Fanon, Albert Memmi, Aimé Césaire, Édouard Glissant et leurs prises de positions anti-coloniales ; Jacques Derrida, Michel Foucault, Gilles Deleuze, Félix Guattari et leur engagement post-structuraliste sont souvent cités comme précurseurs des postcolonial studies. Pascale Rabault-Feuerhahn (2014) parle dans ce sens d'une "dimension transatlantique du postcolonialisme".

"Ensemble hétérogène de travaux de recherche, d'écrits théoriques et d'œuvres littéraires et artistiques [...], procéd[ant] à une critique de l'influence sociale, culturelle, politique et économique, exercée par des groupes sociaux sur des territoires physiques, sur les corps, sur l'imaginaire et les pratiques sociales" (DELLAFAILLE, 2012, p. 15), les études postcoloniales ont une double portée. D'un côté, elles s'intéressent à l'époque coloniale, aux mouvements indépendantistes qui ont entraîné la décolonisation et aux tourments déclenchés lors de la constitution des nouveaux états. ${ }^{6}$ Nous retrouvons ici le sens chronologique ou historique

\footnotetext{
${ }^{6}$ Selon Pierre Boizette, les littératures postcoloniales désigneraient dans ce cas "des littératures nationales dont l'émergence varierait en fonction de l'accession à l'indépendance des pays concernés. Soit, schématiquement, entre le XVIII siècle pour les plus anciens (Haïti, Libéria...) et les années 1970 pour les derniers (Mozambique, Angola, Zimbabwe)" (BOIZETTE, 2013, p. 1).
} 
du mot "post-colonial", où le préfixe "post-" implique une division du temps en trois périodes - anté-coloniale/coloniale/post-coloniale. La particule "post-" indique également "une continuation, un passage d'une époque à une autre (on clôt une étape seulement pour franchir la frontière vers l'étape suivante) et une perpétuation ou une persistance [...] de certains phénomènes, manifestations, liens, etc." (MARCU, sous presse, p. 151). De l'autre côté, les postcolonial studies cultivent une période beaucoup plus vaste, allant de l'époque coloniale jusqu'à de nos jours où l'on essaie de "dépister les traces" (MOURA, 1999, p. 173) de ce passé ineffaçable. Pour Mamadou Diouf, il s'agit d'une investigation simultanée de "l'histoire passée et [des] héritages de la colonisation européenne pour desserrer l'hégémonie économique, intellectuelle et symbolique, dont elle continue de jouir, et pour la réduire à néant" (DIOUF, 2006, p. 26). Le terme "postcolonial", orthographié cette fois-ci sans trait d'union, perd son trait historique. Cela signifie qu'avoir vécu pendant l'époque coloniale n'est plus une condition sine quo none pour être rattaché à cette épistémologie.

À l'heure actuelle, si les études postcoloniales jouissent d'une grande visibilité dans le monde, particulièrement dans l'espace anglophone, elles tentent encore à accéder à une place de choix dans la sphère scientifique française.

Dès leur introduction dans le milieu universitaire français, les postcolonial studies attirent les critiques et les soupçons. Jim Cohen et Maria-Benedita Basto (2008, p. 79) parlent de "résistances ouvertes et quelques tentatives de réappropriation en vue de leur neutralisation". En 2002, soit trois ans après la publication de l'ouvrage Littératures francophones et théorie postcoloniale signé par Jean-Marc Moura - contribution fondatrice de ces théories en France -, Charles Bonn affirmait dans son article "Petit historique d'une réception mouvementée : $\mathrm{du}$ «postcolonial» au «postmoderne»" : "J'ai une certaine réticence face à cette nouvelle théorie américaine à la mode" (BONN, 2002).

Selon Alec G. Hargreaves, trois facteurs expliquent cette émergence attardée et suspicieuse des études postcoloniales en France :

> "les conditions douloureuses dans lesquelles la France s'est séparée de ses colonies. Contrairement à la Grande-Bretagne, qui à partir de l'indépendance de l'Inde en 1947 a liquidé son empire colonial avec relativement peu de violence, la France a tenté de résister à la décolonisation en menant des guerres brutales, coûteuses et 
finalement vaines, d'abord en Indochine, ensuite en Algérie [...]." (HARGREAVES, 2007, p. 26)

> “L'anti-américanisme [...]. Les élites intellectuelles françaises sont habituées à exercer une domination sur la production et la valorisation de la culture à la fois à l'intérieur du pays et à l'extérieur de celui-ci [...]." (HARGREAVES, 2007, p. 26)

> "Le conservatisme des institutions de l'enseignement supérieur et de la recherche [...]. En France, le découpage administratif des études supérieures se prête moins bien à des initiatives pluri-disciplinaires." (HARGREAVES, 2007, p. 27)

Mais, à partir des années 2005, on observe en France une certaine amplification des discussions autour du sujet post(-)colonial. Jim Cohen et Maria-Benedita Basto expliquent cette dynamique

par la convergence de plusieurs controverses publiques, autour d'une question lancinante qui divise l'opinion : celle du rapport entre un passé marqué par la domination coloniale et les rapports sociaux contemporains. Qu'il s'agisse des débats sur la reconnaissance publique de la mémoire de l'esclavage et du colonialisme, sur les moyens de lutte contre la discrimination ethnoraciale, sur les contours de la laïcité et la place de l'islam, ou encore sur la persistance d'une certaine mentalité coloniale envers l'Afrique, c'est chaque fois le poids de l'histoire coloniale française - et européenne - qui est interrogé. (COHEN ; BASTO, 2008, p. 78)

La situation déconcertante des études postcoloniales dans l'espace français et leur caractère hétéroclite ${ }^{7}$ ne favorisent aucunement une analyse minutieuse d'un corpus littéraire périphérique, marqué fortement et à plusieurs échelles par l'hybridité, le métissage, l'extranéité, le "subalternisme", l'entre(-)deux, produit par des auteurs se réclamant de la marge et voulant en même temps échapper à toute catégorisation, désireux d'explorer et de réécrire leur propre histoire, celle de leur famille et de leur communauté, à partir d'une perspective polysémique, postmoderne. La situation est d'autant plus délicate que, pour certains, dans le terme "postcolonial" subsiste encore l'idée de "«colonial», ne serait-ce que

\footnotetext{
${ }^{7}$ N'oublions pas qu'Achille Mbembe (2006, p. 126) les envisage comme "une rivière aux multiples affluents".
} 
phonétiquement" (ALI-BENALI ; SIMASOTCHI-BRONÈS, 2009, p. 58), notion qui évoque un passé commun dont on voudrait bien se défaire (pour des raisons différentes, c'est vrai) - le monde politique, la société française dans son ensemble, ceux qui ont véritablement et douloureusement vécu cette époque, les anciens combattants, les pieds noirs, les harkis et leurs familles. À cela s'ajoutent les "charges sémantiques [...] encore lourdes" (ALI-BENALI ; SIMASOTCHIBRONÈS, 2009, p. 58) du mot postcolonial qui font que la "guerre des mémoires" (ALI-BENALI ; SIMASOTCHI-BRONES, 2009, p. 58) soit loin d'être liquidée. Cela n'empêche que des chercheurs issus d'universités anglophones explorent le corpus beur en s'appuyant sur les apports des postcolonial studies. ${ }^{8}$

En regardant de près les aspects à saisir lorsqu'on essaie de voir si telle ou telle œuvre littéraire est ou non postcoloniale, ${ }^{9}$ nous ne pouvons ne pas nous demander s'il est vraiment si naturel de coller l'étiquette "postcoloniale" à la littérature des intrangers. En nous appuyant dans notre démarche sur deux romans appartenant à ce que nous pourrions appeler "la littérature issue de la mobilité maghrébine", nous constaterons dans ce qui suit que :

\footnotetext{
${ }^{8}$ Alec G. Hargreaves, Voices from the North African Immigrant Community in France: Immigration and Identity in Beur Fiction (1991); Michel Laronde, Autour du roman beur: immigration et identité (1993) ; Alec G. Hargreaves et Mark Mckinney (dir.), Post-Colonial Cultures in France (1997); Michel Laronde, Postcolonialiser la Haute Culture à l'École de la République (2008), etc.

${ }^{9}$ Voilà le portrait de l'auteur postcolonial esquissé par Patrick Sultan dans son ouvrage La scène littéraire postcoloniale: “- L'écrivain accepte la part de lui-même qu'il tient de l'Europe sans la mettre systématiquement en contradiction avec un patrimoine ancestral qu'il revendique sans l'idéaliser. Il ne révère pas non plus le canon littéraire occidental ni la langue qu'il tient des hasards de la colonisation mais les pratique sans prévention ni déchirements.

- Bien que son écriture entretienne un lien profond avec les paysages et les histoires qui l'ont nourrie, il ne collabore pas à la construction d'un grand récit de la nation [...]. Il est souvent un exilé [...], un nomade [...], un expatrié [...]. [Ses œuvres] mettent en cause les notions de frontières, d'identités, d'appartenance.

- Il écrit dans une telle ou telle langue héritée de la colonisation mais sans lui vouer d'amour ou de haine particulière, sans ressentir l'obligation de motiver ce choix". (SULTAN, 2011, p. 51-52, nous soulignons).
} 
> il est quasiment impossible de donner une réponse catégorique à la question "la littérature des intrangers est-elle ou non postcoloniale?"

> toute réponse dépend de l'aspect pris en compte - auteur, statut de la littérature, personnages, thématiques, rapport avec l'Histoire, langue d'écriture, etc.

\section{Orientation postcoloniale du corpus intranger}

Produite par des écrivains nés dans l'Hexagone de parents ayant vécu le phénomène colonial, la littérature issue de l'immigration maghrébine, de même que l'ensemble littéraire produit par les descendants de harkis, se rattache, selon certains chercheurs - Alec G. Hargreaves est peut-être le plus fervent partisan de cette idée -, au corpus postcolonial.

Malgré leur naissance en France, leur vécu indirect de l'époque coloniale et leurs liens insignifiants avec la terre de leurs ancêtres, les auteurs intrangers (issus de l'immigration ou enfants de harkis) sont donc souvent envisagés comme individus "postcoloniaux". Situés à l'intérieur de l'Hexagone, représentant un "nouvel âge" ou une "nouvelle étape" du postcolonialisme (ALBERT, 2005), ils élaborent une littérature correspondant à "l'articulation la plus récente de la mentalité post-coloniale" (LARONDE, 1997, p. 38). "Farouchement hostiles au colonialisme et profondément marqués par les séquelles de celui-ci" (HARGREAVES, 2011, p. 36), ils ne se réclament pas du bled (mythifié) de leurs parents ; bien au contraire, ils "cherchent à se faire valoir à l'intérieur" (HARGREAVES, 2011, p. 37) de la France en tant qu'écrivains tout court, à "démontrer [leur] francité" (HARGREAVES, 2011, p. 37) sans pour autant oublier leurs origines. Leur maintien en marge de la littérature française ${ }^{10}$ est "largement [vécu], selon Hargreaves,

\footnotetext{
${ }^{10}$ L'exclusion du corpus produit par des écrivains qui n'ont immigré de nulle part de la littérature française circulante correspond en effet à un côté métaphorique de "l'illégitimité de la présence chez soi » (BOUAMAMA, 2006, p. 63). Ce que les parents ont vécu avant et après l'Indépendance d'une manière effective, réelle, quotidienne, les enfants - les auteurs intrangers - vivent à la fois d'une manière concrète (discriminations, insultes, délit de faciès, racisme, etc.) et d'une manière "virtuelle" (non-reconnaissance de la qualité esthétique de leurs œuvres littéraires, rattachement du corpus à la nonlittérature, son maintien dans les "banlieues" de la littérature, etc.). Il s'agit donc d' "une filiation" entre le passé colonial et l'époque contemporaine et d' "un héritage" que les parents transmettent à leurs descendants et qui contamine toutes les sphères de leurs vies.
} 
comme un prolongement en France de la domination imposée à leurs parents outre-mer pendant l'époque coloniale" (HARGREAVES, 2011, p. 37). Ce qui fait que l'intérêt de ces auteurs pour le passé colonial des pays de leurs ancêtres soit de plus en plus évident. Ils sont alors préoccupés par le déterrement des traces de la relation coloniale, la restitution de leurs origines, l'affirmation du poids du passé colonial sur leur destin, la valorisation "des parents rendus trop souvent invisibles par leur faible statut social” (HARGREAVES, 2011, p. 40-41), l'exigence de la reconnaissance des phénomènes apparentés au colonialisme dans la société contemporaine (HARGREAVES, 2007, p. 28).

Dans Mohand le harki, Hadjila Kemoum montre qu'il est impossible de se défaire de l'héritage mémoriel fait de bribes de l'histoire familiale ou du pays que les parents ont apportées avec eux en terre d'accueil et qu'ils ont léguées à leurs descendants. L'auteure tente une réécriture personnelle d'un épisode de l'Histoire de l'Algérie - la signature des accords d'Évian qui met fin à la guerre d'indépendance, le départ de l'armée française, l'ascension du FLN et l'impact que tous ces événements ont eu sur la vie de Mohand. Le roman devient ainsi une "écriture du non oubli" (ALI-BENALI ; SIMASOTCHI-BRONÈS, 2009, p. 51) qui s'intéresse "aux conséquences latentes de l'empire qui, comme des parasites de sable logés dans la callosité des pieds" (BOEHMER, 2009, p. 84) surgissent dans l'existence de toute une communauté. Kemoum insiste alors sur les failles du contact non pas entre deux cultures mais entre deux sociétés.

Construit sous la forme d'un journal, Mohand le harki s'offre au lecteur à partir d'un chronotope binaire - l'Algérie et les événements des deux dernières années de la Guerre d'Indépendance ; la France et la vie difficile dans un pays qui a "trahi" les harkis (KEMOUM, 2013, p. 11) (Voir Annexe). Seul Mohand s'inscrit dans les deux espaces-temps ; il n'a jamais parlé à ses enfants de ce passé qui lui "colle à la peau", qui lui "coule dans les veines[,] [qui] dessine [ses] rides, [qui] paralyse [sa] tête et [son] cœur" (KEMOUM, 2013, p. 41), un passé que ses enfants ont "porté" malgré eux (KEMOUM, 2013, p. 41) : "Pour les Arabes, nous étions des «sales harkis». Pour les Français, des bougnoules qui devraient rentrer chez eux" (KEMOUM, 2013, p. 41-42).

Pour ce qui est du roman Un homme, ça ne pleure pas, il manifeste son caractère postcolonial à travers une "affirmation forte de son espace d'énonciation", traduite par la présence de deux "scénographies majeures" 
(BONN, 2006 ; MOURA, 1999) : "une scénographie anthropologique", résultat d'une description susceptible de "crée[r] la cohérence d'un espace périphérique et sa spécificité par rapport à la norme du Centre" (BONN, 2006) ; "une scénographie de la rupture", visible sur le plan de l'écriture.

Les Chennoun sont une famille d'immigrés algériens installés à Nice dans un quartier populaire. Mourad, celui qui narre l'histoire familiale, accepte un poste de professeur de français dans un lycée de la banlieue parisienne. Une fois la scène de l'intrigue déplacée, les descriptions sont plus détaillées : le lecteur pénètre d'un côté dans un quartier chic de Paris, avec des maisons imposantes, aménagées somptueusement, dont les habitants dépensent l'argent sur des choses futiles ; il accède de l'autre côté dans des endroits obscurs ou pauvres, situés dans la banlieue extra ou intra muros de Paris, où se côtoient des individus maudits, désargentés, des personnes issues de l'immigration et des types en mal avec leur existence ou leur statut. Il ne s'agit pas là de la représentation d'un espace inconnu au lecteur, mais d'un espace envisagé souvent comme a-littéraire. La distance symbolique entre la périphérie et le Centre est ici évidente et il est impossible de la faire disparaître (Mourad ne réussit pas à être affecté dans un lycée situé dans un quartier aisé ; Dounia, malgré ses efforts de gommer l'histoire familiale visible sur la couleur de sa peau ou dans la résonance de son nom, incarnera toujours l'image de l'immigré à intégrer, etc.).

En ce qui concerne l'écriture, Faïza Guène fait encore une fois preuve de son talent extraordinaire dans le maniement de la langue parlée transformée en langue littéraire. Les phrases gardent leur caractère rythmé comme à l'oral. Les dialogues des personnages sont simplifiés ce qui laisse au lecteur l'impression d'assister à un échange langagier réel :

"Hé, toi ! Arrête de pleurer ! Reprends-toi ! Tu es un homme ! Merde!

- Et toi! Tu t'es pas vu?!

- Je pleure parce que c'est dur ! C'est trop dur, cette vie de chien.

- C'est dur pour tout le monde ! [...] Allez, ça suffit ..." (GUÈNE, 2014, p. 155)

Afin de créer l'effet d'une langue écrite oralisée, Guène opère des ruptures sur le plan morpho-syntaxique, prosodique, lexical, sémantique et pragmatique. La langue d'écriture se caractérise alors par l'hybridité, concept cher aux études postcoloniales, qui entraîne, "dans 
un espace transculturel, dans un acte transculturel de communication" la "négociation", la "ré-codification" et la "reconstruction" de "l'Autrui, [de] l'Étrangeté et [du] Propre, [du] connu et [de] l'inconnu, [de] l'Hétérogène et [de] l'uniforme" (DE TORO, 2005). L'hybridité devient dans ce cas "un lieu d'énonciation où se négocient la différence ainsi que la pluralité culturelle" (DE TORO, 2005) et, ajoutons-nous, linguistique. Le post-colonialisme n'est plus alors uniquement "une manière de lire, de relire, de ré-écrire le monde, l'histoire, notre vie quotidienne" (DE TORO, 2005), mais aussi une manière de refonder la langue littéraire, de la régénérer.

Bien qu'à une échelle moins importante que le roman Mohand le harki, Un homme, ça ne pleure pas doit aussi son caractère postcolonial à l'héritage mémoriel qu'il convoque. Faïza Guène nous emmène de l'autre rive de la Méditerranée où les Chennoun passent leur vacances ou participent à des fêtes ou à des enterrements; on y retrouve des portraits de gens de là-bas, des chibani ou des vieilles femmes; on y retrouve également la nostalgie des parents, notamment de la mère, qui n'a toujours pas réussi à accepter son immigration et pleure encore son bled, etc.

Les romans de Hadjila Kemoum et de Faïza Guène partagent ensuite un trait important des littératures postcoloniales : 1"'inquiétude incessante [des personnages] sur qui l'on est - en tant qu'individu ou groupe ou communauté" (BHABHA, 2007, p. 17). Mohand, ses enfants (notamment Jacques) d'un côté ; Mourad, Dounia, leurs parents, de l'autre côté, ils sont tous convoqués 'à un exercice de reconstruction de [leur] identité" (KALANGI, 2015, p. 81). Mohand a perdu une partie de soi-même le jour où il a découvert tous les membres de sa famille massacrés par des fellaghas ; il s'aliène encore plus lorsqu'il décide de quitter l'Algérie pour la France afin de protéger sa famille ; par la suite, il plongera de plus en plus dans la souffrance et la solitude car ses enfants ne comprennent pas ses sacrifices, ce que lui a coûté de vivre dans l'Hexagone et lui reprochent constamment de les avoir obligés de rester dans un endroit qui n'est pas le leur. Jacques, celui qui souffre le plus de la "trahison" de la France envers son père, réussira uniquement après la mort de son géniteur à accepter son identité entre-les-deux. Mourad, en choisissant la banlieue parisienne pour le début de sa carrière didactique, essaie en effet de rompre avec la tutelle d'une mère envahissante qui, trop préoccupée de contrôler la vie des autres, leur interdit en effet de vivre véritablement. Dounia, quant à elle, est celle qui provoque la rupture 
dans sa famille ; elle renie son identité, ses origines, l'héritage familial et s'éloigne de tout ce qui peut lui rappeler le fait qu'elle ne s'appelle pas "Christine" ; mais, à la fin du roman, lorsque son père est mort, elle oublie toutes les souffrances que sa mère lui a causées (consciemment ou non). Tous ces personnages doivent accepter donc le rôle essentiel des individus-clefs ou des événements épouvantables dans l'architecture de leur identité et dans le cheminement de leur destinée.

Ajoutons que les personnages-parents des deux romans sont, c'est vrai, à des degrés différents, "des naufragés du natal" (BENSLAMA, 2013, p. 53). Si Mohand ne regarde pas derrière lui avec nostalgie - il avait définitivement choisi la France et a voulu faire de ses enfants des Français ${ }^{11}$ - et que Monsieur Chennoun soit trop enseveli dans son silence pour rendre compte aux autres de ses états d'âme, Milouda et Madame Chennoun vivent, comme d'ailleurs la plupart des personnages-mères des romans des intrangers, une "vie-fantôme", accablées à cause d'un passé perdu à jamais.

Dans Mohand le harki et Un homme, ça ne pleure pas l'empreinte postcoloniale émane aussi des "tensions" entre la culture dans laquelle les personnages-enfants sont nés et la culture dans laquelle leurs parents (notamment les mères) choisissent de continuer de vivre. Jacques et Debhia sont les personnages-contestataires de l'ordre imposé par leurs familles ; ils ne souscrivent pas aux choix ou aux règles adoptés par leurs parents ; ils veulent décider eux-mêmes le trajet de leur vie sans tenir compte des conséquences dévastatrices sur la cohésion de la tribu qu'un tel comportement peut avoir. Ces conflits compliqueront davantage leur reconstruction identitaire, d'autant plus qu'à la fin, tous les deux se rendront compte de l'impossibilité d'effacer l'héritage familial qu'ils portent dans leur âme.

Toujours dans une approche postcoloniale de la littérature issue de la mobilité maghrébine, il est fondamental de s'intéresser à l'héritage linguistique que ce corpus littéraire investit. Dans leurs romans, Kemoum et Guène introduisent ainsi un nombre (assez) important d'épices identitaires pour rendre compte des réalités propres au bled :

\footnotetext{
${ }^{11}$ Les enfants aînés, nés en Algérie, portent des prénoms kabyles - Arezki et Dehbia -, tandis que les cadets, nés en France, portent des prénoms français - Jacques et Christine.
} 
Après les averses d'été, on allait ramasser les escargots et, en fin d'après-midi, à l'intérieur de la mechta, les femmes s'affairaient en cuisine. (GUÈNE, 2014, p. 86)

Un jour, Dounia avait attrapé un crapaud énorme, trouvé au bord de l'oued. (GUÈNE, 2014, p. 88)

- C'est pas pareil! Seigneur! El kebda, el kebda ! (GUÈNE, 2014, p. 101)

Le boujadi insouciant qui menait ses moutons paître dans la montagne est désormais guerrier. (KEMOUM, 2013, p. 50)

Je n'ai pas de réponse, ouldi. (KEMOUM, 2013, p. 48)

Les chibanis, qui n'ont pas perdu une seule miette du court échange entre Mohand et Rachid ou Hamadouche, hochent doucement la tête. (KEMOUM, 2013, p. 47)

Selon Habiba Sebkhi, l'introduction de ces bribes de langue maternelle dans les œuvres des écrivains intrangers montre que la fiction "joue plus particulièrement sur les traces, non sur les assemblages stabilisés ; c'est pourquoi elle relève plus d'une 'pratique remémorante' [ou évocatrice] [...] et non pas «mémorielle»" (SEBKHI, 2000, p. 195).

\section{3 Éclairage postcolonial (parfois) problématique pour la littérature des intrangers}

Tout en redonnant "la voix aux sans voix" (RABAULT-FEUERHAHN, 2014) - trait essentiel des littératures postcoloniales -, la littérature née à la suite de la mobilité maghrébine, avec ses deux sous-divisions - la littérature issue de l'immigration et la littérature des descendants de harkis - n'observe pas toutes les caractéristiques d'un corpus postcolonial.

Tout d'abord, le rapport entre les écrivains intrangers - et Kadjila Kemoum et Faïza Guène ne sont qu'un exemple - et l'époque coloniale est indirect. Ces auteurs n'ont pas vécu réellement la colonisation et, par la suite, la décolonisation, à l'instar des écrivains maghrébins de langue française ayant choisi de s'exprimer en français - langue de la libération de la parole - et, parfois, de s'exiler en France pour des raisons politiques ou personnelles. "L'histoire coloniale" des écrivains nés dans l'Hexagone dans des familles d'immigrés ou de rapatriés ne constitue pas, selon Habiba Sebkhi (2000, p. 177), "une part active dans l'actualité de leur présent. Pour eux [...] il s'agit d'initier un discours de construction et de négociation d'un présent et d'un futur de citoyen". Les deux romans de 
notre corpus ne sont donc pas nés de l'expérience coloniale personnelle; ils ne disent pas la souffrance et la haine des protagonistes contre le colonisateur (Mohand ne s'en prend pas à la France mais à un homme politique qu'il juge responsable de tous ses déchirements; Jacques finit par comprendre que le pays de sa naissance ne lui a jamais été hostile) ; ils ne représentent pas une offensive envers la colonisation (Mohand le harki condamne les massacres abominables commis par le FLN et les fellaghas); Kemoum et Guène, à l'instar des autres représentants de la littérature des intrangers, ne mettent pas en avant le sentiment du deuil, de la séparation, de la rupture, du déplacement sans fin qui traverse comme un fil rouge les œuvres des écrivains maghrébins d'expression française ; les deux auteures n'ont pas eu d'ailleurs tout à reconstruire, à repartir de zéro après avoir tout laissé derrière elles - terre de naissance, êtres chers, langue, une partie de soi-même -, à s'approprier une culture autochtone souvent en décalage avec la culture d'origine ; bien au contraire, elles sont nées en France, ont bénéficié d'une éducation à la française sans pour autant renier leur histoire familiale.

Ensuite, le corpus produit par les écrivains intrangers s'intègre naturellement dans la littérature de langue française intra muros ou hexagonale. Les littératures postcoloniales, par contre, sont souvent définies comme "décentralisées" ou "extra-européennes". ${ }^{12}$ Pour Patrick Sultan, par exemple,

les œuvres postcoloniales diffèrent [...] des œuvres européennes [...]. Elles comprennent [...] comme des parties constitutives, des éléments de culture extra-européenne sans que l'on puisse les dire étrangères aux formes et aux genres en usages dans les littératures européennes. Elles travaillent sur le tissage de plusieurs langues, particulièrement dans un contexte de domination d'une langue par une autre. Leur propos est politique sans qu'elles soient engagées, ou au service d'une cause. [...] Elles manifestent et assument [...] la situation d'ex-colonisé ou de colonisé (sans que leur thématique soit explicitement anti-coloniale) [...]. Ces œuvres sont des réponses [...] à un passé colonial qui a laissé des traces qu'elles explorent et une mémoire qu'elles ravivent. Elles procèdent

\footnotetext{
${ }^{12}$ Voir, par exemple, Bill Ashcroft, Helen Tiffin, Gareth Griffiths, L'empire vous répond. Théorie et pratique des littératures post-coloniales[The Empire Writes Back: Theory and Practice in Post-Colonial Literature], traduit de l'anglais par Martine Mathieu-Job, Jean-Yves Serra, Bordeaux, Presses universitaires de Bordeaux, 2012 [1989].
} 
d'un choc, d'une blessure, d'une douleur, d'une violence, d'un trauma. (SULTAN, 2011, p. 93)

Parler donc dans les mêmes termes d'un côté d'Ahmadou Kourouma, Maryse Condé, Kateb Yacine, Ben Okri, etc., et de l'autre côté de Rachid Djaidani, Magyd Cherfi, Faïza Guène, etc., nous semble faux si l'on pense que les écrivains issus des anciennes colonies françaises évoquent la perte d'un pays, d'une manière de vivre ou d'une langue, tandis que les auteurs issus de l'immigration maghrébine ou descendants de harkis témoignent de leur attachement au pays de leur naissance et à un espace périphérique qu'ils mettent à l'honneur dans leurs œuvres littéraires. Charles Bonn affirme d'ailleurs :

Je ne pense pas que la description littéraire par la théorie postcoloniale puisse véritablement s'appliquer à ces écrivains [issus de l'immigration maghrébine et, ajutons nous, les écrivains descendants de harkis]. Une explication possible serait sans doute à trouver dans le fait que contrairement à leurs aînés les écrivains "maghrébins", ces auteurs souvent éphémères ne se réclament pas d'un espace géographiquement visible de la périphérie colonisée opposée à un centre colonisateur : au contraire, la plupart de leurs textes montrent l'immigration comme partie problématique, mais non-séparable, de la société dite "d'accueil". (BONN, 2005, p. 55)

En fin, dans le cas des écrivains intrangers, le français est une langue naturelle, acquise pendant l'enfance, façonnée par la suite grâce à l'introduction des mots "déguisés" (verlan, argot, mots tronqués), des épices identitaires et générationnelles (MARCU, sous presse), afin qu'elle devienne leur langue d'écriture. Faïza Guène et Hadjila Kemoum ne doivent pas par conséquent "penser la langue" (MOURA, 1999, p. 171) ; elles ne se trouvent donc pas dans une situation d" "insécurité linguistique" (KLINKENBERG, 1996) car, pour elles, la langue française n'est pas "un enjeu autour duquel se cristallisent de nombreuses tensions, de nombreuses angoisses" (LOUVIOT, 2010, p. 41). L'emploi du français, langue maternelle, ne les placent pas "en position d'extériorité" due à une "langue déjà historiquement liée à un patrimoine culturel et littéraire spécifique" (LOUVIOT, 2010, p. 39) ; il ne détermine non plus un "soupçon d'illégitimité" (LOUVIOT, 2010, p. 41) les obligeant à justifier leur choix. 


\section{Conclusion}

Analyser à partir d'une approche postcoloniale le corpus littéraire produit par les écrivains intrangers, issus de l'immigration maghrébine ou descendants de harkis, s'avère une entreprise doublement épineuse. D'un côté, le statut problématique des postcolonial studies en France n'encourage pas (trop) une telle démarche. De l'autre côté, et c'est sur cet aspect que nous avons insisté dans notre contribution, cet embarras est du au caractère hétéroclite des littératures postcoloniales qui permet l'agglomération sous une même étiquette des œuvres littéraires issues d'époques différentes (coloniale/post-coloniale), d'espaces multiples (Europe/hors-Europe), écrites en français langue de l'Autre ou langue naturelle, ce qui fait que les particularités de chaque texte en question soient négligées, pour ne pas dire refoulées.

Dans notre étude, nous avons démontré, à partir des romans Mohand le harki de Hadjila Kemoum et Un homme, ça ne pleure pas de Faïza Guène, qu'il n'est pas du tout évident de donner une réponse ferme à la question "la littérature des intrangers est-elle ou non posctoloniale ?".

Nous avons observé d'abord que l'héritage familial des écrivains, et, ajoutons-nous, des personnages, fait d"'histoires post-coloniales internes à [...] l'identité" (LARONDE, 2008, p. 26), et l'héritage linguistique, qui jaillit dans la langue d'écriture, constituent les arguments principaux pour l'attribution de l'étiquette "postcolonial" au corpus en question. À cela s'ajoutent d'autres problématiques auxquelles Faïza Guène et Hadjila Kemoum, de même que les autres auteurs intrangers, s'intéressent : l'identité hybride toujours à reconstruire, la nostalgie entraînée par le renoncement au pays de naissance, les divergences (voire parfois les ruptures) entre la culture de la matrie et celle du pays d'accueil, etc.

Ensuite, nous avons relevé que, malgré la présence de ces thématiques communes aux littératures postcoloniales, l'histoire personnelle des auteurs - dans notre cas précis de Faïza Guène et de Hadjila Kemoum -, l'espace dont ils se réclament et qu'ils célèbrent dans leurs écrits et leur langue d'écriture nous permettent de douter du caractère proprement postcolonial de la littérature des intrangers.

Il (nous) reste à démontrer que ces réflexions se vérifient (ou non) dans le cas de la littérature issue des autres immigrations (africaine, antillaise, asiatique, etc.). 


\section{Références}

ALBERT, C. L'immigration dans le roman francophone. Paris : Karthala, 2005. Doi : https://doi.org/10.3917/kart.undef.2005.02

ALI-BENALI, Z. ; SIMASOTCHI-BRONÈS, F. Comme une étrange annonce. D'une certaine pratique de la langue à la théorisation du postcolonial. Littérature, [S.1.], n. 154, p. 53-66, 2009.

ALILAT, F. Faïza Guène, ou Candide en banlieue. Jeune Afrique. Paris, 26 oct. 2004. Disponible sur : < http://www.jeuneafrique.com/>. Consultée le : 7 juil. 2018.

BARDOLPH, J. Études postcoloniales et littérature. Paris : Champion, 2001.

BELETRECHE, N. Toxi-cités. Pour en finir avec les ghettos. Paris : Ed. Plon, 2013.

BENESSAIEH, A. La Perspective postcoloniale. Voir le monde différemment. In : O'MEARA, D. ; MCLEOD, A. (Dir.). Théories des relations internationales : contestations et résistances. Montréal : Athéna, Centre d'Études des Politiques Etrangères et Sécurité, 2010. p. 365-378. Disponible sur : $<$ https ://www.researchgate.net/profile/Afef Benessaieh2/publication/280947505_La_perspective_postcoloniale voir_le_monde_differemment/links/55ce2cdb08ae $118 \mathrm{c} 85$ bea597/ La-perspective-postcoloniale-voir-le-monde-differemment $>$. Consultée le : 5 juil. 2018.

BENSLAMA, F. Les Naufragés du natal. In : SEBBAR, L. (Dir.). Le pays natal. Tunis : Ed. Elyzad, 2013. p. 51-58.

BHABHA, H. K. Les Lieux de la culture. Une théorie postcoloniale = The Location of Culture. Trad. Françoise Bouillot. Paris : Payot, 2007.

BOIZETTE, P. Introduction à la théorie postcoloniale. Revue Silène, Paris, 12 oct. 2013. Disponible sur : <http://www.revue-silene.com/f/ index.php?sp=liv\&livre_id=174>. Consultée le : 9 juil. 2018.

BOEHMER, E. Écriture postcoloniale et terreur. Littérature, Paris, n. 154, p. 82-90, 2009. Doi : https://doi.org/10.3917/litt.154.0082 
BONN, C. La Visibilité de l'émigration-immigration dans les littératures maghrébine, française, et de la "seconde génération" de l'immigration : quelle "scénographie postcoloniale" ?. In : GAFAÏTI, H. (Dir.). Migrances, diasporas et transculturalités francophones. Paris : L'Harmattan, 2005. p. 43-56.

BONN, C. Petit historique d'une recéption mouvementée du "postcolonial" au "post-moderne"? Les Ouvrages du CRASC, Oran, 2002. Disponible sur : <https://ouvrages.crasc.dz/index.php/fr/29-le-roman-moderneecriture-de-1\%E2\%80\%99autre-et-de-1\%E2\%80\%99ailleurs/235petit-historique-d $\%$ E2\%80\%99une-r\%C3\%A9ception-mouvement $\%$ C3\%A9e-du-postcolonial-au-postmoderne>. Consultée le : 5 jul. 2018.

BONN, C. Scénographie postcoloniale et ambiguïté tragique dans la littérature algérienne de langue française, ou : pour en finir avec un discours binaire. Communication au Colloque d'Alger et Tipasa. 2006. Dispobible en : <http://www.limag.refer.org/new/index.php?inc $=$ dspart\&art $=00034575>$. Consultée le $: 2$ juil. 2018 .

BOUAMAMA, S. Immigration, colonisation et domination : l'apport d'Abdelmalek Sayad. Contretemps, [S.1.], n. 16, p. 58-67, maï 2006.

COHEN, J. ; BASTO, M.-B. Quelles possibilités pour les études postcoloniales en France ?. Africultures, Paris, n. 72, p. 78-81, 2008.

CONTE, C. Du bon usage des études postcoloniales. Diasporiques, Paris, n. 11, nouvelle série, p. 73-78, sept. 2010.

DE TORO, A. Post-colonialisme - post-colonialité - hybridité. Concepts et stratégies dans la Francophonie el le Maghreb-francophone. Conference a l'Universite Lyon, Lyon, 2005. Disponible sur : <http://www.limag. refer.org/Textes/DeToro/Lyonpostcol2005.pdf>. Consultée le : 9 juil. 2018.

DELLA FAILLE, D. Les Etudes postcoloniales et le "sousdéveloppement". Revue Québécoise de Droit International, Québec, Hors série, p. 11-31, nov. 2012.

DIOUF, M. Les études postcoloniales à l'épreuve des traditions intellectuelles et des banlieues françaises. Contretemps, [S.1.], n.16, p. 17-34, maï 2006. 
FOHR, A. Sagan des cités ou petite sœur de Jamel Debbouze? Le monde selon Faïza. Elle parle le verlan comme le français châtié, raconte le quotidien d'une beurette de banlieue avec humour et acidité. Faïza Guène, 19 ans. Le Nouvel Observateur, Paris, n. 2076, p. 50-51, 2004.

GUENE, F. Faïza Guène : "Mes héros sont des gens ordinaires". CFDT, [S.1.], 27 fév. 2015. Interview à J. Citron. Disponible sur : <https://www. cfdt.fr/portail/actualites/-interview-faiza-guene-mes-heros-sont-des-gensordinaires-srv1_252254>. Consultée le : 9 juil. 2018.

GUÈNE, F. Un homme, ça ne pleure pas. Paris : Fayard, 2014.

HARGREAVES, A. G. Chemins de traverse. Vers une reconnaissance de la postcolonialité en France. Mouvements, Paris, n. 51, p. 24-31, 2007. Doi : https://doi.org/10.3917/mouv.051.0024

HARGREAVES, A. G. Traces littéraires des minorités postcoloniales en France. Mouvements, Paris, Hors-série, "La France en situation postcoloniale?", p. 36-43, sept. 2011.

KALANGI, C. La Théorie postcoloniale et la prospective de l'identité. Pensées Vives, Clermont-Ferrand, n. 1, p. 77-93, 2013.

KEMOUM, H. Mohand le harki. Paris : Éds. Anne Carrière, 2013.

KLINKENBERG, J.-M. L'Aventure linguistique, une constante des lettres belges : le cas de Jean-Pierre Verheggen. Littérature, [S.1.], n. 101, p. 25-39, 1996.

KRAENKER, S. Des écrivains à l'identité hybride, représentants d'une littérature-monde d'aujourd'hui et de demain. Synergies Pays Riverains de la Baltique, Sylvains les Moulins, n. 6, p. 219-227, 2009.

LARONDE, M. Les Littératures des immigrations en France. Question de nomenclature et directions de recherche. Le Maghreb littéraire. [S.1.], v. I. n. 2, p. 25-44, 1997.

LARONDE, M. Postcolonialiser la haute culture à l'École de la République. Paris : L'Harmattan, 2008.

LOUVIOT, M. Poétique de l'hybridité dans les littératures postcoloniales. 2010. $948 \mathrm{f}$. Thèse (Doctorat en Littérature Comparée) - UFR de Lettres Modernes, Université de Strasbourg, Strasbourg Cedex, 2010. 
MARCU, I. La Problématique de l' "entre(-)deux" dans la littérature des “intranger.e.s”. Paris : L'Harmattan. Sous presse.

MBEMBE, A. Qu'est-ce que la pensée postcoloniale ? Entretien, propos recueillis pas Olivier Mongin, Nathalie Lempereur et Jean-Louis Schlegel. Esprit, Paris, n. 330, p. 117-133, déc. 2006.

MOURA, J.-M. La Critique postcoloniale, étude des spécificités. Entretien, propos recueillis par Boniface Mongo-Mboussa. Africultures - Les Mondes en Rélation, Paris, 30 avr. 2000. Disponible sur : $<$ http://www.africultures. $\mathrm{com} / \mathrm{php} /$ ?nav=article\&no=1360>. Consultée le : 7 juil. 2018.

MOURA, J.-M. Littératures francophones et théorie postcoloniale. Paris : Eds. du Seuil, 1999.

MOURA, J.-M. Postcolonialisme et comparatisme. Société Française de Littérature Générale et Comparée, [S.1.], 2006. Disponible sur : $<$ http:// www.vox-poetica.org/sflgc/biblio/moura.html>. Consultée le : 9 juil. 2018.

RABAULT-FEUERHAHN, P. Histoire transculturelle et théories postcoloniales de la littérature. In : (Dir.). Théories intercontinentales. Voyages du comparatisme postcolonial. Paris : Demopolis, 2014. p. 1130. Doi : https://doi.org/10.4000/books.demopolis. 186

SEBKHI, H. Littérature(s) issue(s) de l'immigration en France et au Québec. 2000. 256 f. Thesis (Ph.D) -Faculty of Graduate Studies, University of Western Ontario, Ottawa, 2000.

SUBTIL, M.-P. Faïza Guène, la sale môme qui écrit des best-sellers.Le Monde, Paris, 12 sept. 2006. Livres. Disponible em : $<$ https://www.lemonde. fr/livres/article/2006/09/12/faiza-guene-la-sale-mome-qui-ecrit-des-bestsellers_812185_3260.html>.Consultée le : 7 juil. 2018.

SULTAN, P. La Scène littéraire postcoloniale. Paris : Éds. Le Manuscrit, 2011.

YOUNG, R. Colonial desire : hybridity in theory, culture, and race. Londres ; Nova York : Routledge, 1995. 


\section{Annexe : Le chronotope binaire du roman Mohand le harki de Hadjila Kemoum}

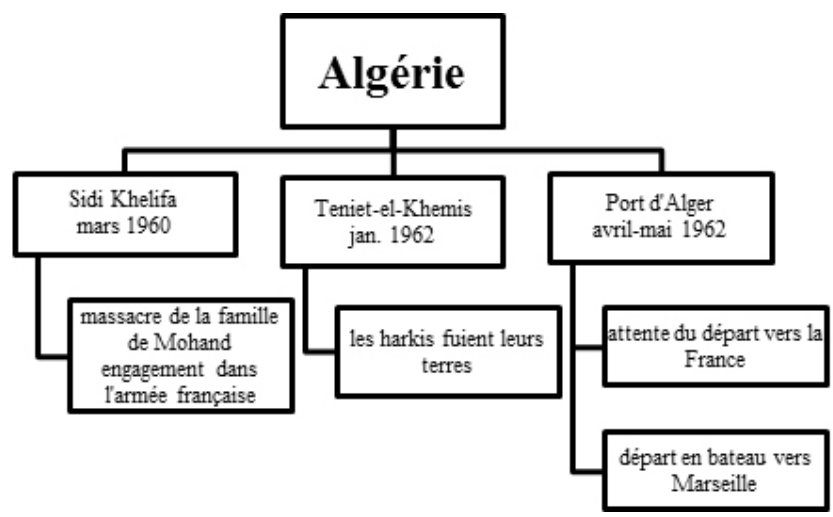

\section{France}

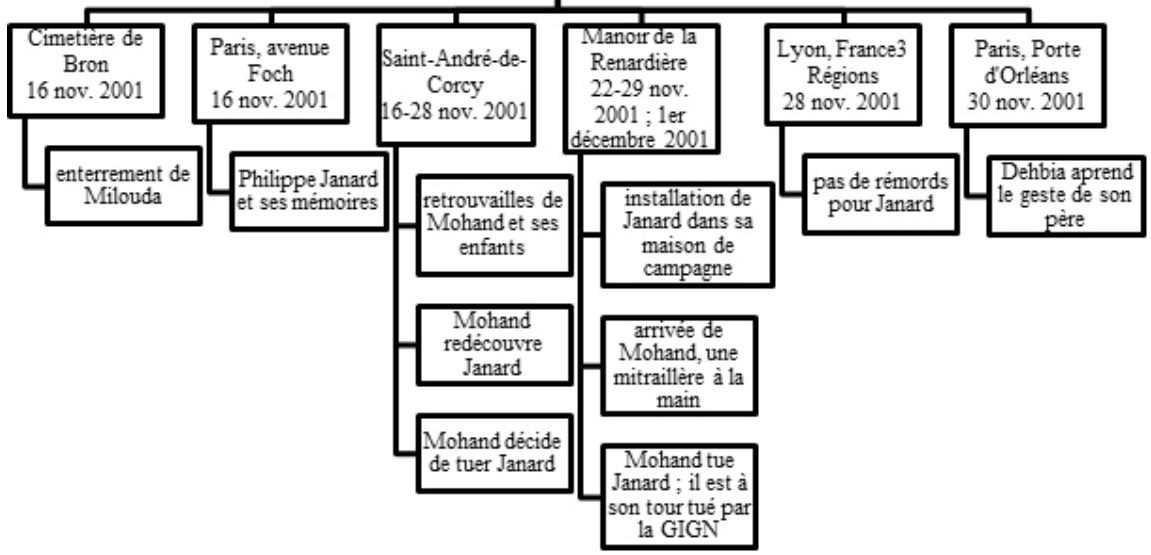

Recebido em: 17 de julho de 2018.

Aprovado em: 2 de agosto de 2018. 
